\title{
EFEITO DO ENSACAMENTO NA QUALIDADE DE MAÇÃS EM DIFERENTES ESTÁDIOS DE DESENVOLVIMENTO ${ }^{1}$
}

\author{
JANAÍNA PEREIRA DOS SANTOS ${ }^{2}$, EDUARDO RODRIGUES HICKEL ${ }^{3}$, \\ LUIZ CARLOS ARGENTA ${ }^{4}$
}

RESUMO-O objetivo deste trabalho foi avaliar a influência do ensacamento na qualidade de maçãs em diferentes estádios de desenvolvimento. O estudo foi conduzido em pomar orgânico, na Estação Experimental da Epagri de Caçador-SC, durante as safras de 2009/2010 e 2010/2011. Os genótipos de macieira avaliados foram 'Royal Gala', 'Fuji Suprema', 'Catarina' e a seleção M-11/00. Os frutos foram avaliados nos estádios de desenvolvimento intermediário (metade do ciclo), na colheita (maturação comercial) e sete dias após a colheita, e em duas diferentes condições (tratamentos): ensacados e não ensacados. O ensacamento foi realizado após o raleio, com embalagens de tecido não texturizado (TNT) de coloração branca, quando os frutos apresentavam em torno de $20 \mathrm{~mm}$ de diâmetro. Os fatores físico-químicos avaliados foram: cor de fundo, diâmetro, massa fresca, firmeza da polpa, índice de amido, acidez titulável e sólidos solúveis totais. Verificou-se que o ensacamento não interferiu na cor de fundo dos frutos nem no teor de sólidos solúveis totais. Entretanto, dependendo do genótipo de macieira e do estádio de desenvolvimento, houve alteração no diâmetro, na massa fresca, na firmeza, no índice de amido e na acidez titulável dos frutos. O ensacamento pode proporcionar frutos maiores e mais pesados, podendo também antecipar a maturação, caracterizada pela redução da firmeza da polpa e pelo aumento do índice de amido. Em alguns genótipos e estádios de desenvolvimento, os valores médios de acidez titulável foram inferiores nos frutos ensacados.

Termos para indexação: Fruticultura de clima temperado, Malus domestica, proteção de frutos, manejo integrado de pragas.

\section{EFFECTS OF BAGGING ON APPLE FRUITS QUALITY UNDER DIFFERENT DEVELOPMENT STAGES}

\begin{abstract}
This study aimed to evaluate the influence of bagging on apple fruit quality on different development stages. The study was conducted in an organic orchard at the Experimental Station of Epagri in Caçador, SC, during the 2009/2010 and 2010/2011 crop seasons. The apple tree genotypes evaluated were 'Royal Gala', 'Fuji Suprema', 'Catarina' and the M-11/00 selection. The fruits were evaluated at the following stages: intermediate development (half-cycle), at harvest or commercial maturity and seven days after harvest. Fruits were submitted to two different conditions (treatments): bagged and no bagged. Fruits were bagged after thinning, at approximately $20 \mathrm{~mm}$ diameter, with white non-textured fabric packaging. The physico-chemical attributes evaluated were: background color, diameter, fresh weight, pulp firmness, starch-iodine index, titratable acidity and total soluble solids. Fruit bagging did not affect fruit background color or total soluble solids. However, fruit diameter, fresh weight, pulp firmness, starch-iodine index and tritratable acidity were altered depending on the apple tree genotype and developmental stage. Bagging can provide larger and heavier fruits, it may also anticipate maturity due to reduction on pulp firmness and increase of the starch-iodine index. In some genotypes and developmental stages the average tritratable acidity values were lower in bagged fruits.
\end{abstract}

Index terms: Temperate-zone fruits, Malus domestica, bagging fruits, integrated pest management.

${ }^{1}$ (Trabalho 016-14). Recebido em: 16-01-2014. Aceito pra publicação em: 26-05-2015.

${ }^{2}$ Eng. Agr., Dr., Pesquisadora da EPAGRI- Estação Experimental de Caçador, Rua Abílio Franco, 1500, Bom Sucesso, C.P. 591, 89500-000, Caçador-SC. E-mail: janapereira@epagri.sc.gov.br

${ }^{3}$ Eng. Agr., Dr., EPAGRI - Estação Experimental de Itajaí. E-mail: hickel@epagri.sc.gov.br

${ }^{4}$ Eng. Agr., Dr., EPAGRI - Estação Experimental de Caçador. E-mail: argenta@epagri.sc.gov.br 


\section{INTRODUÇ̃̃O}

O cultivo da macieira apresenta vários problemas fitossanitários relacionados ao ataque de insetos-pragas, doenças e distúrbios fisiológicos, que podem provocar depreciação na aparência e modificar o sabor da polpa, reduzindo consequentemente o valor comercial dos frutos (SANTOS;WAMSER, 2006). Países importadores da maçã brasileira, tais como Holanda, Reino Unido, Suécia e Alemanha (BITTENCOURT et al., 2011), não têm tolerado resíduos de agrotóxicos nos frutos, levando o setor produtivo a buscar alternativas de manejo. Associado a isso, a preocupação com os riscos à saúde e com os efeitos ambientais indesejáveis tem despertado o interesse de muitos agricultores pela produção orgânica de maçãs (AMARANTE et al., 2008). Em macieiras conduzidas no sistema de produção orgânica, a técnica do ensacamento, na qual os frutos são protegidos individualmente com embalagens de diferentes materiais, vem apresentando resultados promissores na fitossanidade e, consequentemente, na qualidade dos frutos (SANTOS;WAMSER, 2006; SANTOS et al., 2007; TEIXEIRA et al., 2011a,b). Entretanto, além das características externas dos frutos, a qualidade interna também é um fator importante para o consumidor, sendo que esta pode ser afetada por mudanças de microclima causadas pelo ensacamento (WANG et al., 2002). Diferenças nos atributos físico-químicos de frutos submetidos ao ensacamento podem estar relacionadas às características da espécie, cultivar, condições meteorológicas ocorridas em cada safra e do tipo de material da embalagem usada para o ensacamento (TEIXEIRA et al., 2011b). O objetivo deste trabalho foi avaliar a influência do ensacamento na qualidade de maçãs 'Royal Gala', 'Fuji Suprema', 'Catarina' e M-11/00, em diferentes estádios de desenvolvimento.

\section{MATERIAL E MÉTODOS}

O estudo foi conduzido em pomar de macieira cultivado no sistema orgânico de produção, com aproximadamente 0,6 ha e 10 anos de idade, localizado na Estação Experimental da Epagri de Caçador (26 46'30"S e 51 00'54"O), em SC, durante as safras de 2009/2010 e 2010/2011. O pomar é composto por 10 genótipos, cada um disposto em uma linha com aproximadamente 70 plantas, num espaçamento de 4,5 m entre as linhas e 1,5 m entre as plantas. Os genótipos avaliados foram 'Royal Gala', 'Fuji Suprema', 'Catarina' e a seleção M-11/00, todas sobre porta-enxerto Marubakaido com filtro de M-9. Para o controle de plantas invasoras, foram realizadas roçadas, com microtrator nas entrelinhas e com roçadeira costal motorizada entre as plantas. No pomar, não foi realizado tratamento fitossanitário para o controle de insetos. Para o controle de doenças, foram realizadas pulverizações de calda bordalesa e sulfocálcica na concentração de $2 \%$ e $0,15 \%$, respectivamente, de outubro a abril, intercaladas a cada 15 dias, utilizando-se de pulverizador costal. Nas condições climáticas de Caçador, a cultivar Royal Gala apresenta fenologia de maturação semiprecoce, com colheita no início de fevereiro. A seleção M-11/00 apresenta maturação intermediária ou meia-estação, com colheita a partir de meados de fevereiro. As cultivares Fuji Suprema e Catarina apresentam maturação tardia, sendo que a 'Fuji Suprema' é colhida do início a meados de março; e a 'Catarina', de meados de março a início de abril. As avaliações dos frutos foram feitas com base na fenologia de frutificação específica para cada genótipo em estudo, iniciadas a partir da plena floração até à colheita comercial. A plena floração correspondeu à data em que, aproximadamente, $70 \%$ das flores se encontravam abertas. Para verificar o ponto de colheita comercial dos frutos, realizaramse testes para a determinação do índice de amido, conforme Argenta (2006). O ensacamento foi realizado após o raleio, quando os frutos estavam com aproximadamente $20 \mathrm{~mm}$ de diâmetro, 30 dias após a plena floração, no mês de novembro. Os frutos foram ensacados individualmente com embalagens de tecido não texturizado (TNT) de coloração branca (20 $\mathrm{cm} \times 22 \mathrm{~cm}$ e $16 \mathrm{~g} / \mathrm{m}^{2}$ de gramatura). O delineamento experimental utilizado foi completamente ao acaso, com 50 plantas por tratamento. Como margem de segurança, devido às adversidades climáticas, ataque de pássaros e queda natural de frutos, ensacaramse 30 frutos por planta. Para a avaliação da cor de fundo, diâmetro e massa fresca, foram avaliados 100 frutos por tratamento e estádio de desenvolvimento, sendo que cada fruto foi considerado uma repetição. Para a avaliação da firmeza da polpa, do índice de amido, da acidez total titulável e dos sólidos solúveis totais, foram utilizados 50 e 30 frutos, nas safras de 2009/2010 e 2010/2011, respectivamente, sendo que cada fruto foi considerado uma repetição. Assim, dos 30 frutos ensacados por planta, colheram-se dois frutos por planta, por tratamento e estádio de desenvolvimento. Os frutos foram avaliados nos estádios de desenvolvimento: (1) intermediário (na metade do ciclo de desenvolvimento de cada genótipo); (2) colheita comercial (no final do período de crescimento), e (3) sete dias após a colheita comercial, e em duas diferentes condições ou tratamentos: ensacados e não ensacados. A cor de 
fundo dos frutos foi determinada através de escala de cores de 1 a 5, desenvolvida para cultivares dos grupos Gala e Fuji (ARGENTA, 2004a,b). Estas escalas representam a evolução da maturação, em que: (1) indica frutos imaturos, e (5) frutos muito maduros. $\mathrm{O}$ diâmetro transversal $(\mathrm{mm})$ e a massa fresca $(\mathrm{g})$ dos frutos foram medidos com paquímetro digital e balança semianalítica, respectivamente. A firmeza da polpa $(\mathrm{N})$ foi medida com penetrômetro motorizado digital, com êmbolo de $11 \mathrm{~mm}$ de diâmetro. Para tal quantificação, retirou-se uma película da epiderme na região equatorial, nos dois lados opostos, na porção de transição de coloração do fruto. O teor de sólidos solúveis totais foi obtido de pedaços de frutos cortados ortogonalmente, que foram aleatoriamente escolhidos entre os que se realizou a avaliação da firmeza. Com auxílio de uma centrífuga, o suco foi extraído e, através de um refratômetro digital, os valores foram obtidos em graus Brix. A acidez titulável foi expressa em percentagem (\%) de ácido málico, com auxílio de titulador automático digital, através de titulometria de neutralização (com $\mathrm{NaOH} 0,1 \mathrm{~N}$, até pH 8,1). A mesma foi obtida em suco utilizado na avaliação dos sólidos solúveis totais, do qual se pipetaram $10 \mathrm{~mL}$, diluindo-se em $20 \mathrm{~mL}$ de água destilada. O índice de amido foi obtido através de comparação com escalas de escores de 1 a 9, proposta por Bender e Ebert (1985), em que: (1) a superfície corada pelo complexo iodo-amido corresponde à predominância de amido, indicando frutos imaturos, e (9) a superfície não corada corresponde à predominância de açúcares solúveis, indicando frutos totalmente maduros. Os dados foram analisados com auxílio do programa estatístico SPSS versão 18, utilizando-se das rotinas de teste quanto à normalidade, de análise da variância (ANOVA) e de teste F ou de Kruskal Wallis, ambos ao nível de significância de 5\%.

\section{RESULTADOS E DISCUSSÃO}

O ciclo da plena floração até a colheita foi em média de: 107; 124; 146 e 163 dias para 'Royal Gala', M-11/00, 'Fuji Suprema' e 'Catarina', respectivamente. A plena floração ocorreu entre 10 e 20 de outubro, variando de acordo com o genótipo e com as condições meteorológicas da safra avaliada. Verificou-se que o ensacamento não alterou a cor de fundo dos genótipos em nenhum estádio de desenvolvimento dos frutos. No estádio intermediário, o valor médio da cor de fundo foi de, aproximadamente, 2,0, para todos os genótipos e tratamentos (Tabela 1). Já na colheita comercial, verificou-se que os valores variaram de 4,0 a 4,7, indicando que os frutos se encontravam maduros, no ponto ideal para o consumo ou para o armazenamento por curtos períodos (ARGENTA, 2004a,b; ARGENTA et al., 2011). Nos frutos coletados sete dias após a colheita comercial, os valores de cor de fundo não foram superiores a 5,0 em nenhum genótipo e tratamento; o que, de acordo com Argenta (2004a,b), indica que os frutos conservavam a qualidade de textura (Tabela 1), resultados que corroboram os de Teixeira et al. (2011a), que verificaram que a cor de fundo de maçãs 'Imperial Gala' não foi alterada pelo ensacamento com embalagens de TNT. Santos et al. (2007), em estudo realizado com maçãs ensacadas com sacos de papel-manteiga parafinado branco e polipropileno transparente, verificaram que a retirada das embalagens em diferentes épocas não alterou a cor de fundo dos frutos de 'Fuji Suprema' e da seleção M-11/00. Em contrapartida, na cultivar Royal Gala, esses autores verificaram que a permanência das embalagens até a colheita alterou o padrão de cor de fundo, antecipando o amarelecimento, induzindo a colheita dos frutos antes da maturação. No presente estudo, verificou-se que, dependendo do genótipo de macieira e do estádio de desenvolvimento, o ensacamento pode influenciar no diâmetro, massa fresca, firmeza da polpa, índice de amido e acidez titulável dos frutos. Quando ocorreu diferença significativa entre os tratamentos, observou-se que os frutos ensacados foram maiores e mais pesados que os não ensacados (Tabelas 2 e 3). Santos et al. (2007) verificaram que maçãs 'Fuji Suprema' e M-11/00 mantidas ensacadas até a colheita foram maiores e mais pesadas que as não ensacadas. Este fato também foi observado por Grassi et al. (2011), que verificaram que panículas de nespereira ensacadas nos estágios iniciais de desenvolvimento apresentaram maior diâmetro no momento da colheita. Ostapiv et al. (2006), utilizando sacos de polipropileno microperfurado em cachos de uva 'Vênus', verificaram que o ensacamento aumentou o número médio de bagas por cacho, o que favoreceu o aumento da massa média. No presente estudo, o fato de frutos ensacados serem maiores e mais pesados em determinados estádios e genótipos pode ser atribuído aos danos provocados pela mosca-dasfrutas, Anastrepha fraterculus (Diptera: Tephritidae). Isto porque, nos frutos não ensacados, os danos desse inseto chegaram a 97\%; já nos ensacados, foram de até $8 \%$, provavelmente pela oviposição do inseto antes do ensacamento. Santos e Wamser (2006) observaram que maçãs 'Royal Gala' e 'Fuji Suprema', submetidas ao ensacamento, apresentaram menores danos de mosca-das-frutas, além de frutos maiores e mais pesados. Nascimento et al. (2011) 
observaram que, quanto mais precoce o ensacamento de frutos de abieiro, menor o dano causado por mosca-das-frutas, Anastrepha sp. Resultados semelhantes foram obtidos por Teixeira et al. (2011a,b) em maçãs 'Imperial Gala' e 'Fuji Suprema'. O ensacamento também pode antecipar a maturação dos frutos, caracterizada pela redução da firmeza da polpa (Tabela 4) e pelo aumento do índice de amido (Tabela 5). Pereira et al. (2009) observaram que frutos de pinheira ensacados com TNT apresentaram menor firmeza que os não ensacados. Teixeira et al. (2011a) verificaram que maçãs 'Imperial Gala' ensacadas com TNT apresentaram valores do índice de amido superiores às não ensacadas, indicando que o ensacamento acelera a maturação. A aceleração na maturação pode estar relacionada ao aumento da concentração de etileno no interior e no entorno dos frutos ensacados. De acordo com Argenta (2006), a produção e a sensibilidade dos frutos ao etileno variam entre as espécies, e frutos climatéricos, como a maçã, produzem mais etileno que os não climatéricos. Observou-se que o ensacamento alterou o teor de acidez dos frutos, dependendo do genótipo de macieira e do estádio de desenvolvimento, sendo que, quando esta diferença foi observada, menores valores de acidez foram obtidos em frutos ensacados
(Tabela 6). Segundo Wang et al. (2002) e Teixeira et al. (2011b), o aumento da temperatura no interior das embalagens pode contribuir para a redução no conteúdo de ácidos nos frutos. Teixeira et al. (2011b) observaram que a temperatura no interior de embalagens de plástico microperfurado e de TNT, durante o dia, foi superior em 2 a $3{ }^{\circ} \mathrm{C}$ em relação à temperatura de frutos não ensacados. Pedro Júnior (2007) verificou que a proteção individual de cachos de uva 'Romana' provocou alterações de temperatura nas proximidades dos cachos, cujos valores foram de 23,$9 ; 22,9$ e $22,0^{\circ} \mathrm{C}$, para os tratamentos com saco plástico transparente, papel impermeável translúcido (chapéu chinês) e sem proteção, respectivamente. No presente estudo, não se observaram diferenças entre os teores de sólidos solúveis totais, indicando que o ensacamento não alterou este parâmetro (Tabela 7), resultados que corroboram os de Teixeira et al. (2011a,b) para maçãs 'Imperial Gala' e 'Fuji Suprema' também ensacadas com embalagens TNT de coloração branca. O ensacamento também não influenciou no teor de sólidos solúveis totais de frutos em pereira 'Housui' (FAORO; MONDARDO, 2004), em pinheira e atemoieira 'Gefner' (PEREIRA et al., 2009) e em uva 'Romana' (PEDRO JÚNIOR et al., 2007).

TABELA 1 - Valores médios ( \pm DP) da cor de fundo* de maçãs 'Royal Gala', M-11/00, 'Fuji Suprema' e 'Catarina' colhidas em três diferentes estádios de desenvolvimento e em função do ensacamento dos frutos.

\begin{tabular}{lcccc}
\hline \multirow{2}{*}{$\begin{array}{c}\text { Estádio de } \\
\text { desenvolvimento }\end{array}$} & Ensacados & Não ensacados & Ensacados & Não ensacados \\
\cline { 2 - 5 } & \multicolumn{3}{c}{ Royal Gala } \\
\hline Intermediário & $2,0 \pm 0,0 \mathrm{~A}$ & $2,0 \pm 0,0 \mathrm{~A}$ & $2,0 \pm 0,0 \mathrm{~A}$ & $2,0 \pm 0,0 \mathrm{~A}$ \\
Colheita comercial & $4,6 \pm 0,05 \mathrm{~A}$ & $4,5 \pm 0,06 \mathrm{~A}$ & $4,5 \pm 0,06 \mathrm{~A}$ & $4,4 \pm 0,06 \mathrm{~A}$ \\
7 dias após colheita & $4,8 \pm 0,04 \mathrm{~A}$ & $4,5 \pm 0,06 \mathrm{~A}$ & $4,5 \pm 0,05 \mathrm{~A}$ & $4,8 \pm 0,04 \mathrm{~A}$ \\
\hline \multicolumn{5}{c}{ M-11/00 } \\
\hline Intermediário & $2,0 \pm 0,0 \mathrm{~A}$ & $2,0 \pm 0,0 \mathrm{~A}$ & $2,0 \pm 0,0 \mathrm{~A}$ & $2,0 \pm 0,0 \mathrm{~A}$ \\
Colheita comercial & $4,7 \pm 0,05 \mathrm{~A}$ & $4,6 \pm 0,05 \mathrm{~A}$ & $4,3 \pm 0,05 \mathrm{~A}$ & $4,5 \pm 0,05 \mathrm{~A}$ \\
7 dias após colheita & $4,8 \pm 0,04 \mathrm{~A}$ & $4,5 \pm 0,06 \mathrm{~A}$ & $4,8 \pm 0,04 \mathrm{~A}$ & $4,8 \pm 0,04 \mathrm{~A}$ \\
\hline & \multicolumn{5}{c}{ Fuji Suprema } & \\
\hline Intermediário & $2,0 \pm 0,0 \mathrm{~A}$ & $2,2 \pm 0,04 \mathrm{~A}$ & $2,2 \pm 0,04 \mathrm{~A}$ & $2,3 \pm 0,05 \mathrm{~A}$ \\
Colheita comercial & $4,1 \pm 0,06 \mathrm{~A}$ & $4,4 \pm 0,06 \mathrm{~A}$ & $4,4 \pm 0,06 \mathrm{~A}$ & $4,6 \pm 0,05 \mathrm{~A}$ \\
7 dias após colheita & $4,5 \pm 0,06 \mathrm{~A}$ & $4,5 \pm 0,06 \mathrm{~A}$ & $4,8 \pm 0,04 \mathrm{~A}$ & $4,6 \pm 0,05 \mathrm{~A}$ \\
\hline & & Catarina & \\
\hline Intermediário & $2,0 \pm 0,0 \mathrm{~A}$ & $2,0 \pm 0,0 \mathrm{~A}$ & $2,0 \pm 0,0 \mathrm{~A}$ & $2,0 \pm 0,0 \mathrm{~A}$ \\
Colheita comercial & $4,2 \pm 0,04 \mathrm{~A}$ & $4,3 \pm 0,05 \mathrm{~A}$ & $4,3 \pm 0,05 \mathrm{~A}$ & $4,5 \pm 0,05 \mathrm{~A}$ \\
7 dias após colheita & $4,5 \pm 0,06 \mathrm{~A}$ & $4,6 \pm 0,06 \mathrm{~A}$ & $5,0 \pm 0,05 \mathrm{~A}$ & $5,0 \pm 0,05 \mathrm{~A}$ \\
\hline
\end{tabular}

Médias seguidas de mesma letra na linha, dentro de cada safra, não diferem entre si, pelo teste de Kruskal Wallis, a 5\% de probabilidade de erro. *Escala de cores de 1 a 5 para cultivares e seleções dos grupos Gala e Fuji. 
TABELA 2 - Valores médios ( \pm DP) do diâmetro (mm) de maçãs 'Royal Gala', M-11/00, 'Fuji Suprema' e 'Catarina' colhidas em três diferentes estádios de desenvolvimento e em função do ensacamento dos frutos.

\begin{tabular}{|c|c|c|c|c|}
\hline \multirow{2}{*}{$\begin{array}{c}\text { Estádio de } \\
\text { desenvolvimento }\end{array}$} & \multicolumn{2}{|c|}{ Safra 2009/2010 } & \multicolumn{2}{|c|}{ Safra 2010/2011 } \\
\hline & Ensacados & Não ensacados & Ensacados & Não ensacados \\
\hline \multicolumn{5}{|c|}{ Royal Gala } \\
\hline Intermediário & $47,4 \pm 3,75 \mathrm{~A}$ & $47,5 \pm 3,54 \mathrm{~A}$ & $39,9 \pm 6,21 \mathrm{~A}$ & $39,1 \pm 5,81 \mathrm{~A}$ \\
\hline Colheita comercial & $68,3 \pm 4,96 \mathrm{~A}$ & $63,9 \pm 7,97 \mathrm{~B}$ & $70,1 \pm 6,24 \mathrm{~A}$ & $64,9 \pm 8,51 \mathrm{~B}$ \\
\hline 7 dias após colheita & $67,4 \pm 4,81 \mathrm{~A}$ & $65,0 \pm 8,87 \mathrm{~B}$ & $72,8 \pm 5,45 \mathrm{~A}$ & $70,2 \pm 7,43 \mathrm{~B}$ \\
\hline \multicolumn{5}{|c|}{ M-11/00 } \\
\hline Intermediário & $51,0 \pm 4,61 \mathrm{~A}$ & $51,2 \pm 3,42 \mathrm{~A}$ & $50,9 \pm 4,83 \mathrm{~A}$ & $47,4 \pm 5,25 \mathrm{~B}$ \\
\hline Colheita comercial & $66,2 \pm 6,14 \mathrm{~A}$ & $64,2 \pm 5,44 \mathrm{~B}$ & $65,2 \pm 5,24 \mathrm{~A}$ & $59,9 \pm 5,71 \mathrm{~B}$ \\
\hline 7 dias após colheita & $69,0 \pm 5,65 \mathrm{~A}$ & $65,7 \pm 5,91 \mathrm{~B}$ & $68,5 \pm 6,29 \mathrm{~A}$ & $54,2 \pm 6,72 \mathrm{~B}$ \\
\hline \multicolumn{5}{|c|}{ Fuji Suprema } \\
\hline Intermediário & $60,6 \pm 5,21 \mathrm{~A}$ & $60,6 \pm 4,15 \mathrm{~A}$ & $53,9 \pm 6,54 \mathrm{~A}$ & $51,4 \pm 6,71 \mathrm{~B}$ \\
\hline Colheita comercial & $72,7 \pm 5,38 \mathrm{~A}$ & $70,1 \pm 6,64 \mathrm{~B}$ & $65,2 \pm 6,46 \mathrm{~A}$ & $61,1 \pm 7,61 \mathrm{~B}$ \\
\hline 7 dias após colheita & $73,5 \pm 6,44 \mathrm{~A}$ & $72,9 \pm 5,91 \mathrm{~A}$ & $65,4 \pm 5,93 \mathrm{~A}$ & $64,6 \pm 8,84 \mathrm{~A}$ \\
\hline \multicolumn{5}{|c|}{ Catarina } \\
\hline Intermediário & $63,8 \pm 5,30 \mathrm{~A}$ & $61,2 \pm 6,08 \mathrm{~B}$ & $58,8 \pm 7,43 \mathrm{~A}$ & $56,6 \pm 6,27 \mathrm{~B}$ \\
\hline Colheita comercial & $78,5 \pm 7,10 \mathrm{~A}$ & $79,5 \pm 7,39 \mathrm{~A}$ & $75,6 \pm 7,93 \mathrm{~A}$ & $76,1 \pm 6,05 \mathrm{~A}$ \\
\hline 7 dias após colheita & $72,3 \pm 6,54 \mathrm{~A}$ & $74,3 \pm 7,11 \mathrm{~A}$ & $81,0 \pm 10,06 \mathrm{~A}$ & $71,7 \pm 8,48 \mathrm{~B}$ \\
\hline
\end{tabular}

Médias seguidas de mesma letra na linha, dentro de cada safra, não diferem entre si, pelo teste F, a 5\% de probabilidade de erro.

TABELA 3 - Valores médios ( \pm DP) da massa fresca (g)de maçãs 'Royal Gala', M-11/00, 'Fuji Suprema' e 'Catarina' colhidas em três diferentes estádios de desenvolvimento e em função do ensacamento dos frutos.

\begin{tabular}{lcccc}
\hline \multirow{2}{*}{$\begin{array}{c}\text { Estádio de } \\
\text { desenvolvimento }\end{array}$} & \multicolumn{2}{c}{ Safra 2009/2010 } & \multicolumn{2}{c}{ Safra 2010/2011 } \\
\cline { 2 - 5 } & Ensacados & Não ensacados & Ensacados & Não ensacados \\
\hline Intermediário & $49,6 \pm 11,11 \mathrm{~A}$ & $51,4 \pm 9,76 \mathrm{~A}$ & $33,6 \pm 14,38 \mathrm{~A}$ & $32,0 \pm 13,47 \mathrm{~A}$ \\
Colheita comercial & $152,2 \pm 31,91 \mathrm{~A}$ & $126,8 \pm 43,17 \mathrm{~B}$ & $165,1 \pm 41,04 \mathrm{~A}$ & $141,5 \pm 50,71 \mathrm{~B}$ \\
7 dias após colheita & $144,6 \pm 32,90 \mathrm{~A}$ & $135,9 \pm 48,67 \mathrm{~A}$ & $185,7 \pm 40,15 \mathrm{~A}$ & $169,2 \pm 51,15 \mathrm{~B}$ \\
\hline \multicolumn{5}{c}{ M-11/00 } \\
\hline Intermediário & $65,1 \pm 16,57 \mathrm{~A}$ & $65,9 \pm 12,81 \mathrm{~A}$ & $67,4 \pm 17,95 \mathrm{~A}$ & $57,8 \pm 17,43 \mathrm{~B}$ \\
Colheita comercial & $145,6 \pm 35,45 \mathrm{~A}$ & $134,7 \pm 32,37 \mathrm{~B}$ & $145,0 \pm 34,23 \mathrm{~A}$ & $112,0 \pm 33,15 \mathrm{~B}$ \\
7 dias após colheita & $164,1 \pm 38,38 \mathrm{~A}$ & $145,0 \pm 37,65 \mathrm{~B}$ & $151,5 \pm 42,05 \mathrm{~A}$ & $79,8 \pm 29,50 \mathrm{~B}$ \\
\hline & \multicolumn{4}{c}{ Fuji Suprema } \\
\hline Intermediário & $102,8 \pm 24,02 \mathrm{~A}$ & $99,3 \pm 21,11 \mathrm{~A}$ & $72,0 \pm 23,47 \mathrm{~A}$ & $61,7 \pm 21,70 \mathrm{~B}$ \\
Colheita comercial & $174,8 \pm 39,19 \mathrm{~A}$ & $157,3 \pm 41,14 \mathrm{~B}$ & $120,6 \pm 32,32 \mathrm{~A}$ & $98,8 \pm 34,91 \mathrm{~B}$ \\
7 dias após colheita & $185,7 \pm 48,39 \mathrm{~A}$ & $177,2 \pm 43,82 \mathrm{~A}$ & $132,4 \pm 35,08 \mathrm{~A}$ & $131,3 \pm 46,96 \mathrm{~A}$ \\
\hline \multicolumn{4}{c}{ Catarina } \\
\hline Intermediário & $124,9 \pm 32,66 \mathrm{~A}$ & $110,7 \pm 33,01 \mathrm{~B}$ & $92,6 \pm 32,70 \mathrm{~A}$ & $82,6 \pm 26,30 \mathrm{~B}$ \\
Colheita comercial & $233,3 \pm 65,40 \mathrm{~A}$ & $239,5 \pm 71,55 \mathrm{~A}$ & $212,6 \pm 64,44 \mathrm{~A}$ & $209,3 \pm 53,40 \mathrm{~A}$ \\
7 dias após colheita & $179,9 \pm 56,34 \mathrm{~A}$ & $187,9 \pm 51,46 \mathrm{~A}$ & $242,8 \pm 82,28 \mathrm{~A}$ & $170,5 \pm 61,75 \mathrm{~B}$ \\
\hline
\end{tabular}

Médias seguidas de mesma letra na linha, dentro de cada safra, não diferem entre si, pelo teste F, a 5\% de probabilidade de erro. 
TABELA 4 - Valores médios ( \pm DP) de firmeza da polpa (N)de maçãs 'Royal Gala', M-11/00, 'Fuji Suprema' e 'Catarina' colhidas em três diferentes estádios de desenvolvimento e em função do ensacamento dos frutos.

\begin{tabular}{lcccc}
\hline \multirow{2}{*}{$\begin{array}{c}\text { Estádio de } \\
\text { desenvolvimento }\end{array}$} & \multicolumn{2}{c}{ Safra 2009/2010 } & \multicolumn{2}{c}{ Safra 2010/2011 } \\
\cline { 2 - 5 } & Ensacados & Não ensacados & Ensacados & Não ensacados \\
\hline Intermediário & $84,2 \pm 10,29 \mathrm{~A}$ & $82,8 \pm 12,79 \mathrm{~A}$ & $91,8 \pm 11,59 \mathrm{~A}$ & $88,1 \pm 14,44 \mathrm{~A}$ \\
Colheita comercial & $55,9 \pm 11,23 \mathrm{~B}$ & $79,8 \pm 16,18 \mathrm{~A}$ & $56,9 \pm 16,78 \mathrm{~B}$ & $78,5 \pm 19,21 \mathrm{~A}$ \\
7 dias após colheita & $49,2 \pm 8,28 \mathrm{~B}$ & $73,7 \pm 14,63 \mathrm{~A}$ & $52,7 \pm 18,60 \mathrm{~B}$ & $57,5 \pm 16,88 \mathrm{~A}$ \\
\hline \multicolumn{5}{c}{ M-11/00 } \\
\hline Intermediário & $69,6 \pm 8,99 \mathrm{~A}$ & $70,9 \pm 8,99 \mathrm{~A}$ & $65,3 \pm 7,27 \mathrm{~B}$ & $68,9 \pm 10,09 \mathrm{~A}$ \\
Colheita comercial & $65,7 \pm 5,87 \mathrm{~B}$ & $69,8 \pm 7,75 \mathrm{~A}$ & $71,0 \pm 9,65 \mathrm{~B}$ & $81,1 \pm 9,88 \mathrm{~A}$ \\
7 dias após colheita & $62,1 \pm 5,57 \mathrm{~B}$ & $69,7 \pm 8,50 \mathrm{~A}$ & $68,9 \pm 9,63 \mathrm{~B}$ & $80,0 \pm 15,63 \mathrm{~A}$ \\
\hline & \multicolumn{5}{c}{ Fuji Suprema } \\
\hline Intermediário & $59,8 \pm 5,70 \mathrm{~A}$ & $59,6 \pm 5,64 \mathrm{~A}$ & $65,6 \pm 7,29 \mathrm{~B}$ & $69,7 \pm 7,99 \mathrm{~A}$ \\
Colheita comercial & $74,0 \pm 9,22 \mathrm{~A}$ & $75,5 \pm 7,96 \mathrm{~A}$ & $84,7 \pm 10,46 \mathrm{~B}$ & $88,8 \pm 11,58 \mathrm{~A}$ \\
7 dias após colheita & $70,7 \pm 8,17 \mathrm{~A}$ & $69,9 \pm 7,06 \mathrm{~A}$ & $70,7 \pm 8,18 \mathrm{~B}$ & $79,8 \pm 9,72 \mathrm{~A}$ \\
\hline & \multicolumn{5}{c}{ Catarina } \\
\hline Intermediário & $70,9 \pm 7,66 \mathrm{~A}$ & $68,0 \pm 7,08 \mathrm{~A}$ & $70,2 \pm 6,84 \mathrm{~A}$ & $71,5 \pm 7,38 \mathrm{~A}$ \\
Colheita comercial & $77,7 \pm 7,56 \mathrm{~A}$ & $76,2 \pm 10,14 \mathrm{~A}$ & $79,3 \pm 10,92 \mathrm{~A}$ & $82,00 \pm 10,88 \mathrm{~A}$ \\
7 dias após colheita & $78,2 \pm 10,14 \mathrm{~A}$ & $76,8 \pm 9,10 \mathrm{~A}$ & $79,8 \pm 9,35 \mathrm{~B}$ & $84,5 \pm 14,52 \mathrm{~A}$ \\
\hline
\end{tabular}

Médias seguidas de mesma letra na linha, dentro de cada safra, não diferem entre si, pelo teste $\mathrm{F}$, a $5 \%$ de probabilidade de erro.

TABELA 5 - Valores médios ( \pm DP) do índice de amido* de maçãs 'Royal Gala', M-11/00, 'Fuji Suprema’ e 'Catarina' colhidas em três diferentes estádios de desenvolvimento e em função do ensacamento dos frutos.

\begin{tabular}{lcccc}
\hline \multicolumn{1}{c}{$\begin{array}{c}\text { Estádio de } \\
\text { desenvolvimento }\end{array}$} & \multicolumn{2}{c}{ Safra 2009/2010 } & \multicolumn{2}{c}{ Safra 2010/2011 } \\
\cline { 2 - 5 } & \multicolumn{5}{c}{ Ensacados } & Nõo ensacados & Ensacados & Não ensacados \\
\hline Intermediário & $1,0 \pm 0,0 \mathrm{~A}$ & $1,0 \pm 0,0 \mathrm{~A}$ & $1,0 \pm 0,0 \mathrm{~A}$ & $1,0 \pm 0,0 \mathrm{~A}$ \\
Colheita comercial & $8,8 \pm 0,81 \mathrm{~A}$ & $6,4 \pm 3,0 \mathrm{~B}$ & $8,2 \pm 1,72 \mathrm{~A}$ & $5,8 \pm 2,64 \mathrm{~B}$ \\
7 dias após colheita & $9,0 \pm 0,0 \mathrm{~A}$ & $6,7 \pm 2,86 \mathrm{~B}$ & $8,4 \pm 1,83 \mathrm{~A}$ & $8,9 \pm 0,25 \mathrm{~A}$ \\
\hline \multicolumn{5}{c}{ M-11/00 } \\
\hline Intermediário & $1,0 \pm 0,0 \mathrm{~A}$ & $1,0 \pm 0,0 \mathrm{~A}$ & $1,0 \pm 0,0 \mathrm{~A}$ & $1,0 \pm 0,0 \mathrm{~A}$ \\
Colheita comercial & $8,2 \pm 1,28 \mathrm{~A}$ & $7,6 \pm 1,72 \mathrm{~B}$ & $6,3 \pm 2,78 \mathrm{~A}$ & $5,5 \pm 2,61 \mathrm{~A}$ \\
7 dias após colheita & $8,5 \pm 1,15 \mathrm{~A}$ & $8,2 \pm 1,73 \mathrm{~A}$ & $7,3 \pm 2,12 \mathrm{~A}$ & $8,1 \pm 2,01 \mathrm{~A}$ \\
\hline \multicolumn{5}{c}{ Fuji Suprema } \\
\hline Intermediário & $1,7 \pm 1,51 \mathrm{~A}$ & $1,5 \pm 0,73 \mathrm{~A}$ & $1,3 \pm 1,47 \mathrm{~A}$ & $1,0 \pm 0,18 \mathrm{~A}$ \\
Colheita comercial & $7,3 \pm 1,81 \mathrm{~A}$ & $6,0 \pm 2,18 \mathrm{~B}$ & $8,5 \pm 1,06 \mathrm{~A}$ & $8,3 \pm 1,39 \mathrm{~A}$ \\
7 dias após colheita & $7,3 \pm 1,93 \mathrm{~A}$ & $7,1 \pm 1,84 \mathrm{~A}$ & $8,8 \pm 0,66 \mathrm{~A}$ & $8,7 \pm 0,92 \mathrm{~A}$ \\
\hline \multicolumn{5}{c}{ Catarina } \\
\hline Intermediário & $1,8 \pm 1,49 \mathrm{~A}$ & $1,7 \pm 1,06 \mathrm{~A}$ & $1,3 \pm 1,29 \mathrm{~A}$ & $1,2 \pm 0,94 \mathrm{~A}$ \\
Colheita comercial & $7,2 \pm 1,55 \mathrm{~A}$ & $6,6 \pm 1,77 \mathrm{~B}$ & $8,8 \pm 0,63 \mathrm{~A}$ & $8,9 \pm 0,40 \mathrm{~A}$ \\
7 dias após colheita & $8,5 \pm 0,84 \mathrm{~A}$ & $8,2 \pm 1,11 \mathrm{~A}$ & $8,4 \pm 0,96 \mathrm{~A}$ & $8,4 \pm 1,69 \mathrm{~A}$ \\
\hline
\end{tabular}

Médias seguidas de mesma letra na linha, dentro de cada safra, não diferem entre si pelo teste de Kruskal Wallis a 5\% de probabilidade de erro. *Escala de 1 a 9 para cultivares e seleções dos grupos Gala e Fuji. 
TABELA 6 - Valores médios ( \pm DP) da acidez titulável (\% de ácido málico) de maçãs ‘Royal Gala', M-11/00, 'Fuji Suprema' e 'Catarina' colhidas em três diferentes estádios de desenvolvimento e em função do ensacamento dos frutos.

\begin{tabular}{|c|c|c|c|c|}
\hline \multirow{2}{*}{$\begin{array}{c}\text { Estádio de } \\
\text { desenvolvimento }\end{array}$} & \multicolumn{2}{|c|}{ Safra $2009 / 2010$} & \multicolumn{2}{|c|}{ Safra 2010/2011 } \\
\hline & Ensacados & Não ensacados & Ensacados & Não ensacados \\
\hline \multicolumn{5}{|c|}{ Royal Gala } \\
\hline Intermediário & $0,60 \pm 0,012 \mathrm{~B}$ & $0,64 \pm 0,010 \mathrm{~A}$ & $0,62 \pm 0,013 \mathrm{~B}$ & $0,67 \pm 0,0 \mathrm{~A}$ \\
\hline Colheita comercial & $0,30 \pm 0,008 \mathrm{~B}$ & $0,40 \pm 0,010 \mathrm{~A}$ & $0,38 \pm 0,012 \mathrm{~B}$ & $0,43 \pm 0,019 \mathrm{~A}$ \\
\hline 7 dias após colheita & $0,23 \pm 0,024 \mathrm{~B}$ & $0,37 \pm 0,030 \mathrm{~A}$ & $0,32 \pm 0,017 \mathrm{~B}$ & $0,39 \pm 0,016 \mathrm{~A}$ \\
\hline \multicolumn{5}{|c|}{ M-11/00 } \\
\hline Interme & $0,53 \pm 0,030 \mathrm{~A}$ & $0,53 \pm 0,054 \mathrm{~A}$ & $0,50 \pm 0,007 \mathrm{~B}$ & $44 \mathrm{~A}$ \\
\hline Colheita comercial & $0,21 \pm 0,014 \mathrm{~B}$ & $0,25 \pm 0,012 \mathrm{~A}$ & $0,35 \pm 0,004 \mathrm{~B}$ & $0,43 \pm 0,012 \mathrm{~A}$ \\
\hline 7 dias após colheita & $0,20 \pm 0,013 \mathrm{~B}$ & $0,23 \pm 0,009 \mathrm{~A}$ & $0,37 \pm 0,009 \mathrm{~B}$ & $0,39 \pm 0,001 \mathrm{~A}$ \\
\hline \multicolumn{5}{|c|}{ Fuji Suprema } \\
\hline Inter1 & $0,54 \pm 0,006 \mathrm{~B}$ & $10 \mathrm{~A}$ & $0,013 \mathrm{~A}$ & $0,031 \mathrm{~A}$ \\
\hline Colhe & $0,29 \pm 0,007 \mathrm{~B}$ & $0,35 \pm 0$ & $0,37 \pm 0,018 \mathrm{~A}$ & $0,003 \mathrm{~A}$ \\
\hline 7 dias após colheita & $0,31 \pm 0,018 \mathrm{~A}$ & $0,33 \pm 0,014 \mathrm{~A}$ & $0,43 \pm 0,009 \mathrm{~A}$ & $0,43 \pm 0,009 \mathrm{~A}$ \\
\hline \multicolumn{5}{|c|}{ Catarina } \\
\hline Intermedi & $0,62 \pm 0,063 \mathrm{~A}$ & $0,67 \pm 0,003 \mathrm{~A}$ & $0,54 \pm 0,017 \mathrm{~A}$ & $0,55 \pm 0,015 \mathrm{~A}$ \\
\hline Colh & $0,39 \pm 0,176 \mathrm{~A}$ & $0,40 \pm 0,018 \mathrm{~A}$ & $0,48 \pm 0,022 \mathrm{~A}$ & $0,49 \pm 0,024 \mathrm{~A}$ \\
\hline 7 dias após colheita & $0,35 \pm 0,005 \mathrm{~B}$ & $0,41 \pm 0,023 \mathrm{~A}$ & $0,45 \pm 0,009 \mathrm{~A}$ & $0,44 \pm 0,011 \mathrm{~A}$ \\
\hline
\end{tabular}

Médias seguidas de mesma letra na linha, dentro de cada safra, não diferem entre si, pelo teste de Kruskal Wallis, a $\%$ de probabilidade de erro.

TABELA 7 - Valores médios ( \pm DP) de sólidos solúveis totais ('Brix) de maçãs 'Royal Gala', M-11/00, 'Fuji Suprema' e 'Catarina' colhidas em três diferentes estádios de desenvolvimento e em função do ensacamento dos frutos.

\begin{tabular}{|c|c|c|c|c|}
\hline \multirow{2}{*}{$\begin{array}{c}\begin{array}{c}\text { Estádio de } \\
\text { desenvolvimento }\end{array}\end{array}$} & \multicolumn{2}{|c|}{ Safra 2009/2010 } & \multicolumn{2}{|c|}{ Safra $2010 / 2011$} \\
\hline & Ensacados & Não ensacados & Ensacados & Não ensacados \\
\hline \multicolumn{5}{|c|}{ Royal Gala } \\
\hline Intermediário & $8,2 \pm 0,16 \mathrm{~A}$ & $8,2 \pm 0,11 \mathrm{~A}$ & $7,3 \pm 0,20 \mathrm{~A}$ & $7,5 \pm 0,15 \mathrm{~A}$ \\
\hline Colheita comercial & $13,5 \pm 0,30 \mathrm{~A}$ & $13,2 \pm 0,31 \mathrm{~A}$ & $12,8 \pm 0,10 \mathrm{~A}$ & $12,2 \pm 0,0 \mathrm{~A}$ \\
\hline 7 dias após colheita & $13,0 \pm 0,21 \mathrm{~A}$ & $12,8 \pm 0,12 \mathrm{~A}$ & $13,0 \pm 0,0 \mathrm{~A}$ & $13,0 \pm 0,12 \mathrm{~A}$ \\
\hline \multicolumn{5}{|c|}{ M-11/00 } \\
\hline Intermediário & $9,1 \pm 0,61 \mathrm{~A}$ & $9,7 \pm 0,42 \mathrm{~A}$ & $8,3 \pm 0,10 \mathrm{~A}$ & $8,4 \pm 0,20 \mathrm{~A}$ \\
\hline Colheita comercial & $12,7 \pm 0,10 \mathrm{~A}$ & $12,4 \pm 0,15 \mathrm{~A}$ & $12,6 \pm 0,10 \mathrm{~A}$ & $12,8 \pm 0,12 \mathrm{~A}$ \\
\hline 7 dias após colheita & $12,9 \pm 0,13 \mathrm{~A}$ & $13,3 \pm 0,34 \mathrm{~A}$ & $13,5 \pm 0,12 \mathrm{~A}$ & $13,3 \pm 0,12 \mathrm{~A}$ \\
\hline \multicolumn{5}{|c|}{ Fuji Suprema } \\
\hline Intermediário & $9,2 \pm 0,13 \mathrm{~A}$ & $9,1 \pm 0,31 \mathrm{~A}$ & $9,0 \pm 0,00 \mathrm{~A}$ & $9,7 \pm 0,37 \mathrm{~A}$ \\
\hline Colheita comercial & $13,4 \pm 0,48 \mathrm{~A}$ & $13,6 \pm 0,30 \mathrm{~A}$ & $14,4 \pm 0,47 \mathrm{~A}$ & $14,3 \pm 0,29 \mathrm{~A}$ \\
\hline 7 dias após colheita & $14,2 \pm 0,10 \mathrm{~A}$ & $14,6 \pm 0,19 \mathrm{~A}$ & $14,4 \pm 0,20 \mathrm{~A}$ & $14,6 \pm 0,27 \mathrm{~A}$ \\
\hline \multicolumn{5}{|c|}{ Catarina } \\
\hline Intermediário & $9,6 \pm 0,34 \mathrm{~A}$ & $9,5 \pm 0,27 \mathrm{~A}$ & $9,8 \pm 0,19 \mathrm{~A}$ & $10,0 \pm 0,07 \mathrm{~A}$ \\
\hline Colheita comercial & $15,4 \pm 0,19 \mathrm{~A}$ & $15,3 \pm 0,09 \mathrm{~A}$ & $14,5 \pm 0,29 \mathrm{~A}$ & $14,6 \pm 0,30 \mathrm{~A}$ \\
\hline 7 dias após colheita & $14,8 \pm 0,52 \mathrm{~A}$ & $14,7 \pm 0,19 \mathrm{~A}$ & $14,6 \pm 0,0 \mathrm{~A}$ & $13,9 \pm 0,23 \mathrm{~A}$ \\
\hline
\end{tabular}

Médias seguidas de mesma letra na linha, dentro de cada safra, não diferem entre si, pelo teste de Kruskal Wallis, a $5 \%$ de probabilidade de erro. 


\section{CONCLUSÕES}

O ensacamento de maçãs 'Royal Gala', 'Fuji Suprema', 'Catarina' e M-11/00, com embalagens de TNT de coloração branca, não interfere na cor de fundo e no teor de sólidos solúveis totais. Dependendo do genótipo de macieira e do estádio de desenvolvimento, o ensacamento pode proporcionar frutos maiores e mais pesados, diminuir os valores médios de acidez titulável e antecipar a maturação dos frutos.

\section{AGRADECIMENTOS}

Os autores agradecem a Empresa Brasileira de Pesquisa Agropecuária - EMBRAPA, pela concessão de bolsa de Pós-Graduação ao primeiro autor e ao Núcleo de Análise Estatística da Universidade Federal do Rio Grande do Sul - NAE/UFRGS, pelo auxílio nas análises estatísticas.

\section{REFERÊNCIAS}

AMARANTE, C.V.T.; STEFFENS, C.A.; MAFRA, A.L.; ALBUQUERQUE, J.A. Yield and fruit quality of apple orchards under conventional and organic production systems. Pesquisa Agropecuária Brasileira, Brasília, v.43, n.3, p.333-340, 2008.

ARGENTA, L.C. Fisiologia pós-colheita: maturação, colheita e armazenagem de frutos. In: EPAGRI (Ed.). A cultura da macieira. Florianópolis: GMC/Epagri, 2006. cap.20, p.691-732.

ARGENTA, L.C. Índice de cores para maçãs "Fuji”. Florianópolis: Epagri, 2004a. 1p.

ARGENTA, L.C. Índice de cores para maçãs “Gala". Florianópolis: Epagri, 2004b. 1p.

ARGENTA, L.C.; VIEIRA, M.J.; SCOLARO, A.M.T. Validação de catálogos de cores como indicadores do estágio de maturação e do ponto de colheita de maçã. Agropecuária Catarinense, Florianópolis, v.23, n.3, p.71-77, 2011.

BENDER, R.J.; EBERT, A. Determinação do ponto de colheita de cultivares de macieira. Teste iodoamido. Florianópolis: Empasc, 1985. 6p.
BITTENCOURT, C.C.; MATTEI, L.F.; SANT'ANNA, P.R. de; LONGO, O.C.; BARONE, F.M. A cadeia produtiva da maçã em Santa Catarina: competitividade segundo produção e packing house. Revista de Administração Pública, Rio de Janeiro, v.45, n.4, p.1199-1222, 2011.

FAORO, I.D.; MONDARDO, M. Ensacamento de frutos de pereira cv. Housui. Revista Brasileira de Fruticultura, Jaboticabal, v.26, n.1, p.86-88, 2004.

GRASSI, A.M.; SCARPARE FILHO, J.A.; CHAGAS, E.A.; PIO, R.; PASQUAL, M.; TIZATO, L.H.G.; CHAGAS, P.C. Qualidade de frutos de cultivares de nespereira em função do ensacamento em diferentes estádios de desenvolvimento. Ciência Rural, Santa Maria, v.41, n.2, p.227-229, 2011.

NASCIMENTO, W.M.O.; MÜLLER, C.H.; ARAÚJO, C.S.; FLORES, B.C. Ensacamento de frutos de abiu visando à proteção contra o ataque da mosca-das-frutas. Revista Brasileira de Fruticultura, Jaboticabal, v.33, n.1, p.48-52, 2011.

OSTAPIV, F.; MAZARO, S.M.; DONAZZOLO, J.; CITADIN, A.; LINK, M.; GOUVÊA, A. Influência do ensacamento sobre a qualidade da uva 'Vênus'. Synergismus Scyentifica, Pato Branco, v.1, p.6469, 2006.

PEDRO JÚNIOR, M.J.; PEZZOPANE, J.R.M.; HERNANDES, J.L.; LULU, J.; DE CASTRO, J.V. Avaliações microclimáticas e das características de qualidade da uva de mesa 'Romana' com proteção individual de cachos. Bragantia, Campinas, v.66, n.1, p.165-171, 2007.

PEREIRA, M.C.T.; BANDEIRA, N.; ANTUNES JÚNIOR, R.C.; NIETSCHE, S.; OLIVEIRA JÚNIOR, M.X.; ALVARENGA, C.D.; DOS SANTOS, T.M.; OLIVEIRA, J.R. Efeito do ensacamento na qualidade dos frutos e na incidência da broca-dos-frutos da atemoieira e da pinheira. Bragantia, Campinas, v.68, n.2, p.389-396, 2009.

SANTOS, J.P.; WAMSER, A.; DENARDI, F. Qualidade de frutos ensacados em diferentes genótipos de macieira. Ciência Rural, Santa Maria, v.37, n.6, p.1614-1620, 2007. 
SANTOS, J.P.; WAMSER, A.F. Efeito do ensacamento de frutos sobre danos causados por fatores bióticos e abióticos em pomar orgânico de macieira. Revista Brasileira de Fruticultura, Jaboticabal, v.28, n.2, p.168-171, 2006.

TEIXEIRA, R.; AMARANTE, C.V.T.; BOFF, M.I.; RIBEIRO, L.G. Controle de pragas e doenças, maturação e qualidade de maçãs 'Imperial Gala' submetidas ao ensacamento. Revista Brasileira de Fruticultura, Jaboticabal, v.33, n.2, p.394-401, 2011 a.
TEIXEIRA, R.; BOFF, M.I.C.; AMARANTE, C.V.T.; STEFFENS, C.A.; BOFF, P. Efeito do ensacamento dos frutos no controle de pragas e doenças e na qualidade e maturação de maçãs 'Fuji Suprema'. Bragantia, Campinas, v.70, n.3, p.688695, $2011 b$.

WANG, S.M.; GAO, H.J.; ZHANG, X.B. Effects of bagging on pigment, sugar and acid development in 'Red Fuji' apple fruits. Acta Horticulturae, The Hague, v.29, p.263-265, 2002. 